\title{
Depression in Temporal Lobe Epilepsy: A Review of Prevalence, Clinical Features, and Management Considerations
}

\author{
C. S. Garcia \\ Department of Psychiatry, Schulich School of Medicine, University of Western Ontario, London, ON, Canada N6A 5C1 \\ Correspondence should be addressed to C. S. Garcia, Carla.Garcia@lhsc.on.ca
}

Received 4 July 2011; Accepted 10 September 2011

Academic Editor: Warren T. Blume

Copyright () 2012 C. S. Garcia. This is an open access article distributed under the Creative Commons Attribution License, which permits unrestricted use, distribution, and reproduction in any medium, provided the original work is properly cited.

Depression in temporal lobe epilepsy has been established as a frequent occurrence, and various possible mechanisms for this significant comorbidity have been posited. However, there is still little to guide a clinician in the recognition and management of depression in patients with temporal lobe epilepsy. This is in part due to the lack of consistent findings in earlier studies, which was likely partly due to variabilities in methodology, sampling, and diagnosis of both temporal lobe epilepsy and depression. However, in recent years, significant effort has been made to address these issues and provide a framework for diagnosis and management of depression in this population. The following is a review of the literature, with special emphasis on clinical phenomenology of depressive symptoms, described bidirectional risk between depression and temporal lobe epilepsy, and treatment strategies in the context of potential drug interactions with antiepileptic drugs.

\section{Introduction}

Temporal lobe epilepsy (TLE) is the most frequent of the epileptic disorders. Interictal depressive symptoms, and interictal major depressive episodes, are quite common in epilepsy in general but appear to be particularly linked to TLE [1]. This link between these two disorders has been a source of great interest to both neurologists and psychiatrists for many years and has generated an expansion of knowledge in both fields that has been used to better understand not only these two disorders, but the relationship of mood, cognition, and temporolimbic function in other related conditions as well [1].

Despite this, a review of the literature reveals that there is limited cohesive guidance regarding the prevalence of depression in TLE patients and the clinical features by which diagnosis can be made, nor are there universally accepted guidelines for the management of depression in this population. Difficulties in study design, variable sample populations, and the challenges of confirming temporal lobe focus in some patient populations are all possible contributors to this gap. However, many established authorities in this field have been making significant efforts to overcome these issues and move towards a cohesive approach to diagnosis and management of depression in the TLE population.

\section{Prevalence of Depression in Temporal Lobe Epilepsy}

Although there have been many studies examining the frequency of depression in temporal lobe epilepsy patients, it has been difficult to establish a clear pattern with respect to prevalence, particularly in comparison to other types of epilepsy [2]. Historically, depressive symptoms have been considered to be more frequent in epilepsy with a temporal lobe focus than in extra-TLE or generalized epilepsy [3-8]. However, several other studies have not been able to document any such differences [2, 9-17].

Several explanations for this have been proposed. Firstly, concomitant or additive mechanisms may play a role in the development of depressive symptoms in sufferers of TLE. Rodin et al. [7] noted that many patients with TLE have more than one seizure type, and that the number of seizures rather than the location of the focus may be more relevant. Two later studies confirmed that frequency of seizures were more 
significant in predicting depressive symptoms than focus location $[18,19]$. Other risk factors have also been identified, such as age of onset and laterality of temporal lobe focus that may also place patients at higher or lower risk than location of focus alone [20,21]. Similarly, frontal lobe dysfunction in addition to temporal lobe dysfunction may be an important risk factor in developing depression in TLE patients [2]. Additionally, Swinkels et al. [2] described significant methodological differences among the various studies examining prevalence rates of depression in epilepsy, including small sample size, lack of control groups, variable and often nonstandardized diagnostic instruments, and variability in the study population (inpatients, outpatients, surgical patients, etc.). Finally, Adams et al. [15] observed that many studies assessing psychiatric symptoms in epilepsy predate the advent of technology such as video electroencephalogram monitoring and magnetic resonance imaging, which may make characterization of the underlying lesion more unreliable. All of the above may be contributing to the fact that although there is a plethora of studies dedicated to the assessment of prevalence of depression in temporal lobe epilepsy, consensus has yet to be achieved.

Nevertheless, a handful of recent studies have attempted to rectify some of the above issues and have provided some interesting data. Sanchez-Gistau et al. [16] studied 308 patients that were carefully classified as having epileptiform foci that was temporal or extratemporal in localization. These patients were then administered the Structured Interview for DSM-IV Axis I Psychiatric Disorders (SCID-I), and results were compared using a multivariate analysis. Of the TLE group, $22 \%$ had a lifetime prevalence of Major Depressive Disorder, and $14.6 \%$ had previous one-year prevalence of this disorder. Extra-TLE sufferers had a lifetime prevalence of $14.6 \%$ and a previous one-year prevalence of $6.5 \%$. Of all the psychiatric disorders assessed (mood disorders, psychotic disorders, somatoform disorders, substance abuse disorders, and anxiety disorders), only previous-year prevalence of major depressive disorder was significantly different between the two groups, with TLE sufferers having a higher rate of major depression. Adams et al. [15] followed 319 individuals with focal epilepsy over an 11-year period. The epileptic site, laterality, and type of the lesion were confirmed with video electroencephalogram monitoring and MRI scans. The patients were assessed by the study neuropsychiatrist using DSM IV diagnostic criteria prior to their epilepsy diagnosis being made and were reviewed by a second neuropsychiatrist to confirm diagnostic concordance. The overall prevalence of depression in the study participants was $32.6 \%$. No significant differences were found in the prevalence of depression between TLE patients (31.2\%) and extra-TLE patients $(37.9 \%)$, nor between left or right-sided lesions. Of note, patients with nonlesion focal epilepsy exhibited a higher rate of depression $(41.6 \%)$ compared to those with a lesion on MRI, regardless of temporal or extratemporal focus. Swinkels et al. [17] assessed 67 TLE patients and 64 extratemporal lobe focus patients for depression using the Beck Depression Inventory and the Composite International Diagnostic Interview. No significant differences were noted between the two localization groups, but rates of psychiatric symptoms were higher in patients with more frequent and prolonged seizures.

In summary, the question of whether a temporal lobe focus of epilepsy assigns a higher prevalence of depression to patients is not yet fully answered. Earlier studies were divided on this issue, but variability in methodology, patient samples, accuracy of identification of epileptic focus, and in diagnostic tools used for assessment of depression all made comparison of the data difficult. Additionally, this variability in results may also represent multiple factors at play in the development of depression beyond simply localization of epileptic focus, such as frequency and length of seizures, age, and comorbid dysfunction across multiple areas.

\section{Clinical Characteristics of Depression in Temporal Lobe Epilepsy}

Although this survey of the available literature did not uncover studies of the natural history of depressive symptoms in TLE patients specifically, most studies examining the relationship between depressive symptoms and age of onset of epilepsy or duration of epilepsy do not identify clear association with risk of depression $[8,22,23]$. One study did find a potential link between depression and development of seizures in later life [24].

One of the questions that has been raised historically is whether depressive symptoms in the epilepsy and TLE population represent a comorbid mood disorder, with diagnostic characteristics and a natural history similar to major depressive disorder as seen in the general population, or whether these symptoms instead represent a collection of emotional and cognitive disabilities similar but not equal to a major depressive disorder [25-27].

The concept of interictal dysphoric disorder was proposed to describe the latter in response to studies suggesting that a significant portion of epilepsy patients with depressive symptoms would not have met criteria for Major Depressive Disorder as typically described in diagnostic schedules [28, 29]. For example, Kanner et al. [30] examined patients with refractory seizures and depressive symptoms and found only 29\% met DSM IV criteria for Major Depressive Disorder. Initially proposed by Kraepelin, then later Bleuler, this concept originally described a pattern of symptoms consisting of prominent irritability, euphoria, anxiety, anergia, insomnia, and pain. These symptoms are described to have a chronic, relapsing and remitting course, but to respond well to antidepressants [31]. A more specific range of symptoms had been described by Blumer et al. in their Neurobiological Inventory for Epilepsy, which was a reformulation of earlier inventories that were meant to define the TLE personality characteristics $[25,29]$. Two categories of symptoms have been described: depressive-somatoform symptoms (depressed mood, anergia, pain, and insomnia) and affective symptoms (irritability, euphoric mood, fear, and anxiety) [29]. Anhedonia has also been proposed as a better marker for depression in patients with epilepsy, in part secondary to its independence from physical symptoms associated with medications and chronic illness [32]. 
However, there have also been many proponents of the belief that there is not sufficient evidence to support a model of psychopathology unique to temporal lobe epilepsy. Kanner and Nieto [33] proposed that the symptoms described in these TLE- or epilepsy-specific psychopathology inventories are quite similar to a stable mood disorder with marked depressive and anxiety features rather than a de novo condition. Lishman [34] also concurred that the depressive symptoms described as specific to the TLE population were an artifact of sampling and selective reporting by patients in institutional settings.

Efforts have been made to test the validity of diagnosing depression in this population. Reilly et al. [25] reasoned that the latent variable factors observed to be impacted in depressive disorders (negative attitude, performance difficulty, and somatic elements) could be measured in TLE patients to compare the level of dysfunction across these domains to known quantities in major depression. TLE patients manifested difficulties across these domains that were very similar to that seen in major depressive disorder, suggesting that these symptoms represented a major depressive disorder, rather than a condition unique to TLE. Jones et al. documented the validity of the SCID and MINI (Mini International Neuropsychiatric Interview) by comparing to patient self-report of symptoms of Major Depressive Disorder, and finding very high concordance [35].

In summary, there has been some suggestion historically that depressive symptoms in the TLE population may represent not depression but rather a condition unique to these patients. This concept has been described as interictal dysphoric disorder. However, there now also have been studies that appear to confirm that the DSM IV criteria for major depression are valid in the TLE population, and that the depressive symptoms they experience can be understood as a stable mood disorder.

\section{Neuroanatomical Findings of Depression in Temporal Lobe Epilepsy}

The various structures of the limbic system have been a focus of interest in understanding both depression and TLE for quite some time. Regions of particular interest for both these disorders include the temporal lobes (particularly the hippocampus, amygdala, entorhinal, and neocortical cortex), the frontal lobes, and important limbic subcortical structures such as the basal ganglia and thalamus, as well as the circuits connecting all these structures $[1,37-40]$. Although the hippocampus and amygdala have been the major focus of attention, all of the above structures have been found to share association across these two disorders [1].

In both depression and TLE, hippocampal volumes have been found to be abnormal [1]. In depressed patients with and without TLE, hippocampal volumes are reduced, usually bilaterally or occasionally left sided only [37, 41-45]. In TLE patients, volumes are usually reduced on the side of the epileptic focus [40, 42, 45]. In depressed TLE patients, hippocampal volumes are usually reduced bilaterally. Interestingly, in patients with a left-sided TLE focus, cognitive problems with memory and learning are more marked [46]. Other studies have confirmed this, linking depression and verbal learning impairments in TLE patients [47]. The suspected cause behind this is dysfunction within the larger language representation in the left hemisphere [46, 47].

The amygdala has also been a source of intense study in the combined field of depression and TLE, given its key role in fear and associated emotions [48]. This structure appears to change as an acute depressive episode becomes chronic, initially becoming enlarged bilaterally, then shrinking bilaterally as the mood disorder becomes chronic [1]. Two studies have found a relationship between escalating amygdala volume and severity of symptoms of depression in the TLE population $[49,50]$. Left-sided volume increases of the amygdala and severity of depression symptoms in TLE patients seemed particularly associated with each other [50]. The suspected mechanism for this is hyperactivity of the amygdala in the acute phase of depression in TLE and non-TLE patients, resulting in an increase in volume secondary to increased regional blood flow $[1,49,50]$.

The two commonest lesions for the development of temporal lobe epilepsy are mesial sclerosis and the more rare neocortical temporal lesions [1]. Patients with mesial temporal sclerosis have significantly higher rates of depression than those with neocortical temporal lesions, regardless of lateralization [51]. Additionally, patients with mesial temporal sclerosis have a greater frequency of cognitive side effects and mood problems with antiepileptic drugs [52]. Interestingly, Salgado et al. [53] found significantly more widespread grey matter volume loss in TLE patients with depression as compared to their nondepressed fellow patients. This leads to the suggestion that there is a bidirectional relationship between these two disorders [53].

In summary, various important structures of the limbic system have been found to be significantly different in depressed TLE patients in comparison with nondepressed fellow patients. There may be a bidirectional relationship between depression and TLE influencing these structures.

\section{Depression after Neurosurgery in Temporal Lobe Epilepsy}

Surgery for intractable epilepsy has become increasingly available for patients, resulting in more individuals becoming seizure-free, often thus dramatically improving quality of life $[35,54]$. However, there is an emerging recognition that psychiatric complications can occur in the postoperative period, including de novo symptoms of depression [54].

The strongest risk factor for depression in the postoperative course is, perhaps not surprisingly, preoperative depression [55-57] and has been reported in approximately 20 $30 \%$ of patients undergoing surgery [58-60]. Rates of de novo depression in TLE patients in the postoperative period range from 5 to $25 \%$ [54]. Other risk factors identified include older patients at time of surgery [61, 62], male gender [55], strong family history of psychiatric illness, and poor seizure outcome postoperatively [61]. The highest risk period appears to be in the first 3 months following surgery, with slow improvement at the 12- or 24-month mark [54, 63]. 
Lateralization has been the focus of many studies, but no clear, cohesive pattern appears to have emerged yet. Several studies suggest that right temporal lobe resections represent a greater risk of postoperative depression $[57,64]$, while several more support left temporal lobe resection as higher risk for this complication $[56,57]$, and yet others report no evidence of laterality at all [63]. These ambivalent results are echoed in the literature examining rates of depression following tumor resections $[65,66]$.

However, some studies have documented what appears to be a bidirectional risk in the relationship between postoperative seizure control and depressive symptoms. Metternich et al. [67] documented significantly lower Beck Depression Inventory scores in patients that were seizure-free postoperatively. Reuber et al. [58] observed that postoperative TLE patients improved significantly with respect to depressive symptoms in comparison to medically managed TLE patients, but only if seizure control was significantly improved. This led them to suppose that depressive symptoms were associated with epileptic activity rather than structural changes.

Finally, Wrench et al. [68] recruited 60 patients undergoing two types of surgery (mesial temporal lobe resection and nonmesial temporal lobe resection) and followed them longitudinally. Preoperatively, $43 \%$ of these patients had a lifetime prevalence of depression, with no difference between the surgical groups. Predictive factors for preoperative depression included family history of mental illness and financial dependency. However, in the postoperative phase, the mesial temporal resection group experienced a significantly higher rate of depression, both recurrence and de novo [68], suggesting that perhaps disruption of these structures carries a higher risk of depression as a complication postoperatively.

In summary, recurrence and de novo development of depression is a risk in TLE patients undergoing surgery, particularly in the first 3 months postoperatively. Although no clear pattern is emerging regarding the relevance of laterality, there is significant evidence to support a bidirectional relationship between depression and postoperative seizure control, where the presence of one can exacerbate the other. This echoes the findings previously described earlier in this chapter, in which each condition may operate as a significant risk factor for the other.

\section{Antiepileptic Drugs and Depression}

Antiepileptic drugs (AED's) have been known to have positive psychotropic effects beyond their antiseizure effect for quite some time [69]. Indeed, many AED's have separate indications for the treatment of psychiatric disorders, including roles as mood stabilizers [70], anxiolytics [71], and in the management of withdrawal syndromes [72]. It is also equally true that many AED's have negative psychotropic effects that can complicate the management of both epilepsy and depression in patients [69].

The AED's associated with the highest risk of occurrence of depressive symptoms in patients with epilepsy are those which act at the benzodiazepine-GABA receptor complex [69]. These include barbiturates, topiramate, and vigabatrin.
Levetiracetam and felbamate appear to represent an intermediate risk of depressive symptoms, leaving the other AED's as either low risk or unknown [69] (Table 1). Unfortunately, a clear understanding of which populations may be at particular risk of developing depressive symptoms while on AED's has yet to be developed [69], and this review of the literature did not produce any studies that endeavored to identify this in the TLE population. However, Mula and Schmitz [69] suggests a general approach of monotherapy if possible, with introduction of any new AED's with slow, careful titration, and careful histories of premorbid and family psychiatric disorders being collected regularly in this patient population [69].

\section{Consequences of Depression in Temporal Lobe Epilepsy}

7.1. Depression as a Risk Factor for Seizures. Depression has been acknowledged by the World Health Organization as one of the most significant sources of burden of disease and suffering globally [73]. The impact of this disorder on mortality, morbidity, quality of life, social function, and occupational function have been well described. Similarly, the additive burden of depression in chronically medically ill people has also been well described, both for epilepsy as well as other medical conditions as diverse as COPD, ESRD, cancer, and diabetes. Thus, it is expected that there would be a consequence to be borne by those TLE patients that carry the comorbidity of these two disorders.

However, as previously noted in this chapter, there is strong support for a bidirectional risk existing between these two disorders. In other words, the presence of depression may have a direct impact on TLE symptom severity, control, and possibly even onset.

For example, Forsgren and Nystrom [74] found that there was a seven-fold increase in rates of depression being diagnosed prior to the onset of the seizure disorder in patients with newly diagnosed epilepsy when compared to ageand sex-matched controls. This remarkable finding was further raised to a 17 -fold increase when patients with a localized onset were studied. Another study found a 3.7-fold increase in frequency of diagnosis of depression preceding the first seizure in older adults with new onset epilepsy [24]. A study of Icelandic children and adults with new onset epilepsy found a similar increase in rates of depression preceding seizure onset ( 1.7 fold), as well as a 5.1-fold increase in a premorbid history of attempted suicide [75]. In a study of children with new onset seizures, psychopathological symptoms (including anxiety, depression, attention disorders, thought disorders, and somatic disorders) were present at higher rates than controls for $32 \%$ of the newly epileptic children [76]. Finally, recent studies have also suggested that psychopathology could be a significant risk factor for infants developing nonfebrile seizures or epilepsy in childhood [77].

Perhaps the most interesting finding of the pattern of mood symptoms predating epilepsy is Alper et al's. [78] study of epileptic patients enrolled in SSRI, SNRI, and mirtazepine treatment trials. Patients on the medications had significantly lower rates of seizures when compared to their 
TABLE 1: Positive and negative effects of AED's [36].

\begin{tabular}{|c|c|c|}
\hline $\begin{array}{l}\text { Antiepileptic } \\
\text { drug }\end{array}$ & $\begin{array}{l}\text { Negative psychotropic } \\
\text { symptoms }\end{array}$ & $\begin{array}{l}\text { Positive psychotropic } \\
\text { symptoms }\end{array}$ \\
\hline Barbiturates & $\begin{array}{l}\text { Depression, } \\
\text { hyperactivity }\end{array}$ & Anxiolytic, hypnotic \\
\hline $\begin{array}{l}\text { Carbamazepine/ } \\
\text { oxcarbazepine }\end{array}$ & Irritability & $\begin{array}{l}\text { Mood stabilizer, } \\
\text { antimanic }\end{array}$ \\
\hline Ethosuximide & $\begin{array}{l}\text { Behavioral } \\
\text { abnormalities, } \\
\text { psychosis }\end{array}$ & None identified \\
\hline Felbamate & $\begin{array}{l}\text { Depression, anxiety, } \\
\text { irritability }\end{array}$ & None identified \\
\hline Gabapentin & $\begin{array}{l}\text { Behavioral problems } \\
\text { in children }\end{array}$ & None identified \\
\hline Lamotrigine & Insomnia, agitation & $\begin{array}{l}\text { Mood stabilizer, } \\
\text { antidepressant }\end{array}$ \\
\hline Levetiracetam & $\begin{array}{l}\text { Irritability, emotional } \\
\text { lability }\end{array}$ & Possible antimanic \\
\hline Phenytoin & Encephalopathy & Possible antimanic \\
\hline Pregabalin & Unknown & Anxiolytic \\
\hline Tigabine & $\begin{array}{l}\text { Depression } \\
\text { (nonconvulsive status } \\
\text { epilepticus) }\end{array}$ & Possible anxiolytic \\
\hline Topiramate & $\begin{array}{l}\text { Depression, } \\
\text { psychomotor slowing, } \\
\text { psychosis }\end{array}$ & Mood stabilizer \\
\hline Valproate & Encephalopathy & Mood stabilizer \\
\hline Vigabatrin & $\begin{array}{l}\text { Depression, } \\
\text { aggression, psychosis }\end{array}$ & None identified \\
\hline Zonisamide & $\begin{array}{l}\text { Agitation, depression, } \\
\text { psychosis }\end{array}$ & Possible antimanic \\
\hline
\end{tabular}

matched fellow patients receiving placebo. This last study in particular is suggestive of the potentially exacerbating role that untreated depressive symptoms may have on seizure control.

7.2. Suicidality in TLE Patients. Completed suicide is one of the most tragic and feared outcomes of a depressive episode and is always a concern to clinicians when working with patients with significant psychiatric comorbidity. Fortunately, it is a relatively rare event in the general population. Unfortunately, in epilepsy, the rate of suicide is approximately two to five times that of the general population, and this is further elevated to a 25-fold increase among patients with TLE $[79,80]$. Not surprisingly, the rate of completed suicide is further elevated up to 32 -fold by the presence of a comorbid depressive disorder [79].

This shocking increase has been considered to be primarily influenced by the psychosocial consequences of living with chronic epilepsy [81]. However, recent data would suggest that the situation is more complex than psychosocial consequences of chronic illness alone, and that a part of understanding completed suicide risk in epilepsy may lie in the examination of suicide attempts. A study comparing suicide attempts among patients with epilepsy to comparably handicapped controls with other chronic disabilities found that $30 \%$ of patients with epilepsy had attempted suicide as compared to $7 \%$ of controls $[80,81]$. This is relevant given the fact that suicide attempts are complex behaviors involving many factors, including impulsivity and executive dysfunction, which can be associated with temporolimbic function [82-86]. Jones et al. [87] found a lifetime prevalence of suicide attempts of $20.8 \%$ among 139 outpatients followed at epilepsy centers in the United States. In this sample, the highest rates of attempts were among patients with a lifetime history of a major depressive episode or manic episode, and higher rates of suicidal ideation were also associated with a lifetime history of mood or anxiety disorders. Major depressive disorder was the most frequent psychiatric disorder identified among patients with a history of suicide attempt $(51.7 \%)$, while anxiety disorders were more strongly associated with suicidal ideation (58.8\%) in this sample [87].

This suggests that there are characteristics specific to epilepsy, in particular TLE, which may place patients at elevated risk of completed suicide and suicide attempts, particularly in the context of comorbid depression. Espinosa et al. [88] studied 42 patients with newly diagnosed TLE over a 1-year period and assessed suicide risk via the Plutchik Risk of Suicide scale. They found that $57.1 \%$ of their sample scored $>7$ on this scale, which is the highest risk category for suicide, $28.6 \%$ had a past history of suicide attempts, and $45.2 \%$ had experienced suicidal thoughts. The study authors also assessed for multiple associated factors including psychiatric comorbidity, past medical and psychiatric history, and neuropsychological deficits. They discovered a significant relationship between higher suicide risk and a higher rate of suicide attempts in patients with a family history of psychiatric diseases, left-sided TLE, current major depressive episode, and higher perseverative responses on neuropsychological testing via Wisconsin Card Sorting Test (WCST). They also found a strong correlation between poor WCST performance and severity of depressive symptoms, implying that the presence of depression likely exacerbates executive dysfunction in a population that already has documented difficulty in this area. Once again, this suggests a bidirectional relationship between depression and epilepsy in the manifestation of suicide risk.

There is an added layer of complexity in understanding suicidality in TLE patients. On December 16, 2008, the US Food and Drug Administration issued a warning about an increased risk of suicidal ideation and behavior among people taking AED's [89]. The agency had performed a review of 119 clinical trials of 11 AED's and noted a 1.8-fold increase in suicidal behavior or ideation in patients taking AED's in comparison to those taking placebo. Although many methodological issues have been identified with this study, it raised concerning questions regarding the used of these drugs in a population known to suffer significant psychiatric comorbidity. As described previously in this chapter, AED's have been documented to produce both positive and negative psychotropic effects, and the FDA's announcement produced a flurry of epidemiologic work to attempt to confirm this increased risk of suicidality and hopefully suggest mechanisms [90]. These questions are still being answered, and the studies' findings have been quite mixed, but results thus far 
suggest that if there is an elevated risk of suicidality with AED's, it appears to be very low [69], and that no clear pattern is emerging regarding risk stratification across the various AED's $[69,90]$. Additionally, none of these studies have focused on TLE in particular, but rather have included samples that tend to be quite broad both in type of epilepsy and comorbidity of psychiatric illness [90], which in part may explain the variability of findings. Wen et al. [90] noted an interesting trend in their retrospective analysis of patients in the Comprehensive Epilepsy Research Program (CERP) database over a 32-month period. Briefly, they noted that the strongest predictors for the development of suicidality over time were the presence of depressive symptoms or suicidality prior to AED treatment, and that those patients started on new or multiple AED's had less improvement of suicidality over time in comparison with those who had no changes made to their AED regimen. Additionally, they found no significant difference in suicidality between the AED's themselves. This led the authors to suggest that perhaps the findings of the original FDA study were generated by the artifact of patients on AED's improving less than placebo controls with respect to their suicidality symptoms over time.

\subsection{Quality of Life Consequences for Patients with Comorbid} Depression and TLE. Multiple studies in epilepsy in general and TLE specifically have made efforts to examine the relationship between various factors associated with living with epilepsy and the impact on quality of life [91-98]. Kanner [99] reviewed 5 studies in particular that consistently demonstrated that depression was the most powerful predictor of health-related quality of life across multiple domains, even when controlling for factors such as seizure frequency, severity, and other psychosocial variables. Meldolesi et al. [98] studied 106 patients with drug-resistant unilateral temporal lobe epilepsy, administering various standardized quality of life instruments as well as the Beck Depression Inventory and an anxiety scale. They also found depression to be consistently the strongest predictor of lower scores on all QOL domains except seizure worry. This effect was independent of socioeconomic status, gender, lateralization of seizure focus, seizure frequency and severity, and anxiety.

Additionally, comorbid depression appears to be associated with a greater likelihood of adverse events associated with antiepileptic drugs, more frequent visits to physicians, and higher cost of medical care related to the seizure disorder, rather than any cost associated with the psychiatric disorder and its treatment [97-99]. This is an interesting finding in light of the recurring theme woven through the literature of the bidirectionality of depressive symptoms and epilepsy symptoms. Again, the presence of one appears to make the management of the other more of a challenge.

\section{Management Strategies for Depression in Temporal Lobe Epilepsy}

In general, evidence for treatment strategies of mood disorders in epilepsy are lacking, and development of management approaches tend to rely on clinical experience rather than evidence-based trials favoring one treatment over another [69]. The paucity of data is even more pronounced when examining the literature for TLE-specific depression treatment studies. Not surprisingly then, there are no widely accepted guidelines for the treatment of depression in TLE patients.

However, clinicians can turn to a body of literature that, while lacking in large, double-blinded, and placebo-controlled RTC's, still includes several smaller open label trials, case series reports, and a handful of comparative studies. The bulk of this data does not limit itself to TLE although a recently published comparative study in TLE patients in particular is included in the following discussion.

8.1. Antidepressants. There are three main considerations when initiating an antidepressant trial in a patient with epilepsy: exacerbation of seizure control, potential for interaction with AED's, and efficacy of the antidepressant in depression symptom resolution. There appears to be variability between and also within the various antidepressant drug classes although some generalizations can be made from the available literature.

8.1.1. Antidepressants and Seizure Risk. The potential for antidepressants to provoke seizures has been a source of concern and possibly a barrier to treatment of depression in patients with epilepsy [100]. The data documenting seizures secondary to antidepressants is derived largely from psychiatric populations, in vitro or animal model studies, or from samples which were not specifically patients with epilepsy $[36,100]$. Often, seizures associated with antidepressants are described in cases of toxicity, such as accidental or intentional overdose $[36,100]$. This makes it difficult, if not impossible, to generalize findings to epilepsy patients. The situation is further complicated by the fact that animal and human studies suggest that some antidepressants may have an anticonvulsant effect, while some may have a proconvulsant effect, and yet others may have a biphasic effect, in which they are anticonvulsant at lower doses and proconvulsant at higher doses [36]. Mechanisms proposed for the proconvulsant effect include the anticholinergic effect of many antidepressants (particularly at higher doses), as well as the elevation of serotonin and noradrenalin neurotransmission [36]. Anticonvulsant effect may be mediated by the interaction of the antidepressant with other factors, including AED's. For example, fluoxetine has been noted to enhance the anticonvulsant effect of phenytoin and carbamazepine via selective inhibition of serotonin uptake [36].

Tricyclic antidepressants (TCA's) have a wide variety of neurotransmitter related effects, many of which are dose dependent as well. Overall, they are considered proconvulsant, in large part secondary to their significant anticholinergic effect, which is known to lower seizure threshold [36]. As stated previously, some selective serotonin reuptake inhibitors (SSRI's) may actually have an anticonvulsant effect [101103]. In general, clinical and research experience suggests that the risk of seizures with SSRI's is very low and perhaps not different from placebo, and certainly lower than with 
TCA's [101]. A special consideration with SSRI's is the fact that they can promote hyponatremia, which can represent a risk in the precipitation of seizures [36]. Other antidepressants which are considered to be "high risk" for seizures in the general population or in toxicity studies (e.g., bupropion) have been found overall to have an acceptably low risk when prescribed correctly [36].

Overall, the incidence of seizures with antidepressants is less than $0.5 \%$, particularly when used within the recommended therapeutic range and when other risk factors are excluded [36] (Table 2). Given the significant consequences described earlier in this chapter associated with depression in TLE, it would therefore seem that concerns about exacerbating seizure control should not be enough to rule out antidepressants in most TLE patients.

8.1.2. Interactions between Antidepressants and AED's. TCA's have, by nature of their longevity, perhaps the most collective clinical experience in many medical conditions, epilepsy included. Amitriptyline, clomipramine, and imipramine are extensively metabolized by CYP 1A2, 2D6, and 3A4 (Table 3). Nortriptyline and desipramine, the metabolites of amitriptyline and imipramine respectively, are metabolized mainly by CYP 2D6. All AED's with enzyme-inducing properties (barbiturates, carbamazepine, and phenytoin) all induce the metabolism of TCA's. However, carbamazepine also reduces protein binding of imipramine and desipramine, resulting in increased free fraction of these drugs, and thus resulting in little need to alter dosing of these two TCA's when coadministered with carbamazepine. Valproate can inhibit the metabolism of TCA's resulting in increased plasma levels at comparable dosage. Overall, coadministration of TCA's and AED's can be done safely and effectively as long as dosing is done slowly and carefully and is accompanied by regular monitoring of blood levels of both drugs [36].

SSRI's are generally more tolerable and safe than TCA's, due in part to their reduced anticholinergic effect and inability to block sodium channels, even in overdose [104]. As a result, these drugs are widely prescribed. Fluoxetine and paroxetine are metabolized by CYP 2D6, sertraline by CYP 3A4, fluvoxamine by CYP $1 \mathrm{~A} 2$, and finally citalopram by CYP $2 \mathrm{C}$ [104]. Paroxetine is an inhibitor of CYP 2D6, fluvoxamine inhibits CYP 1A2, and fluoxetine moderately inhibits CYP 2D6 and 3A4. Sertraline and citalopram do not seem to have significant induction/inhibition properties [105].

Fluoxetine has a high risk of interaction with phenytoin, but less clearly with carbamazepine [36, 106, 107]. Paroxetine and sertraline seem to have low risk of interaction with phenytoin $[36,106]$. However, carbamazepine seems to induce citalopram's metabolism significantly, thus reducing plasma concentrations and possibly efficacy of this drug [36]. Paroxetine appears to have little interaction with carbamazepine, valproate, or phenytoin [36]. Fluvoxamine appears to have no effect on carbamazepine levels [36]. However, there is a paucity of evidence on the safety of fluvoxamine coadministration with valproate or phenytoin [36]. Sertraline was found to increase the levels of lamotrigine in two documented cases [36]. Finally, sertraline was found to have no
TABLE 2: Seizure prevalence in psychiatric samples during treatment with antidepressant drugs [36].

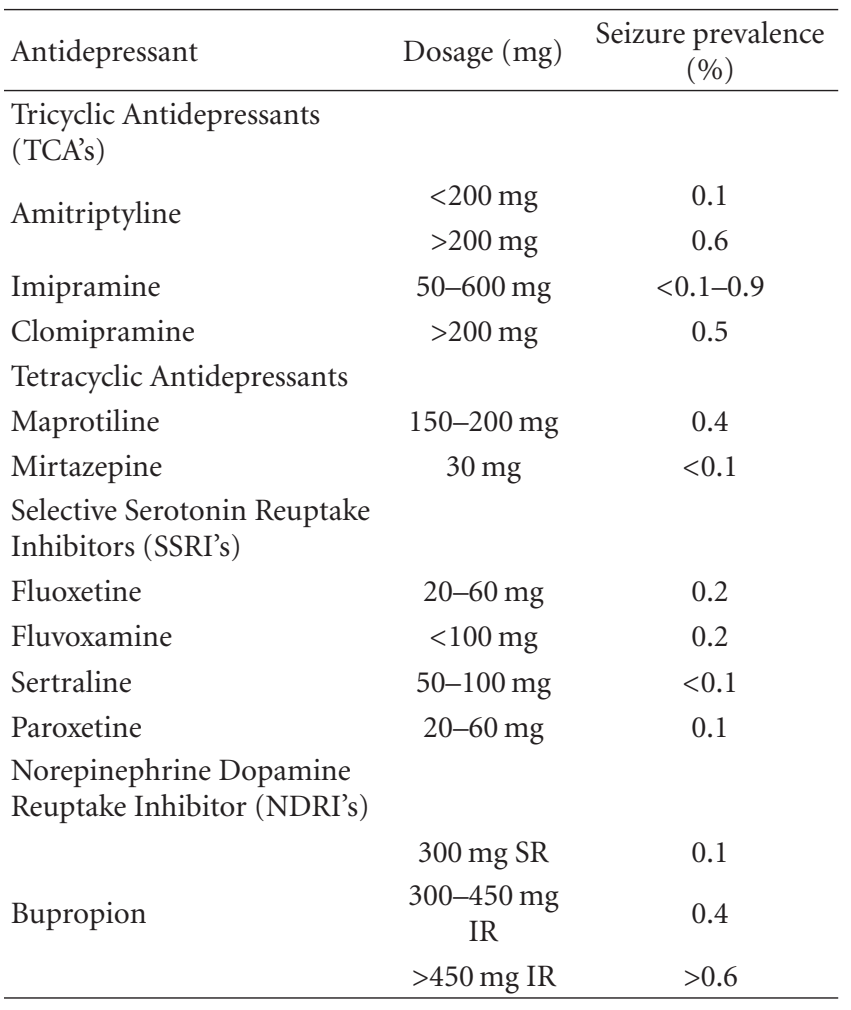

significant effect on carbamazepine levels in a double-blind randomized, placebo-controlled trial of 14 healthy volunteers [36]. Sertraline and clonazepam were also found to be safe in coadministration [36].

SNRI's (venlafaxine and duloxetine) are primarily metabolized by CYP 2D6 [104], but interactions with AED's have yet to be studied [36].

Mirtazepine is a noradrenergic and specific serotonergic reuptake inhibitor (NaSSA) and is primarily metabolized by CYP 2D6. Studies are lacking in this population on interactions with AED's, but this medication has been noted to bind histaminic receptors, resulting in sedation, increased appetite, and weight gain. Given that these are side effects of many AED's, the potential for an additive effect is present [36].

Bupropion is a noradrenergic and dopaminergic reuptake inhibitor (NDRI). Carbamazepine is a potent inducer of the metabolism of bupropion, significantly reducing plasma levels [104]. Bupropion in turn has marked inhibitory properties, increasing levels of valproate and phenytoin [36].

8.1.3. Efficacy of Antidepressants in TLE. Again, there is a dearth of evidence-based, controlled trials that attempt to study efficacy of antidepressants in epilepsy in general, and the number of trials specific to TLE found in this review of the literature was a single one.

The earliest controlled trial in patients with epilepsy involved amitriptyline and an antidepressant that no longer exists (nomifensine). At 6 weeks, improvement of depressive symptoms was documented in both drugs, but at 12 weeks, 
TABLE 3: CYP450 enzymes inhibition and induction by antidepressants and antiepileptic drugs [36].

\begin{tabular}{|c|c|c|c|}
\hline $\begin{array}{l}\text { CYP } \\
\text { isoenzyme }\end{array}$ & Inhibitors & Inducers & Substrates \\
\hline \multirow[t]{5}{*}{ CYP 1A2 } & Fluvoxamine & St. John's Wort & Antidepressants \\
\hline & Fluoxetine & & TCA's \\
\hline & Paroxetine & & Fluvoxamine \\
\hline & Tertiary TCA’s & & Mirtazepine \\
\hline & & & Duloxetine \\
\hline \multirow[t]{7}{*}{ CYP 2C9/10 } & Fluoxetine & Phenobarbital & Antidepressants \\
\hline & Fluvoxamine & Carbamazepine & Sertraline \\
\hline & & Phenytoin & Fluoxetine \\
\hline & & & Amitriptyline \\
\hline & & & Bupropion \\
\hline & & & Antiepileptics \\
\hline & & & Phenytoin \\
\hline \multirow[t]{14}{*}{ CYP 2C19 } & Fluvoxamine & Carbamazepine & Antidepressants \\
\hline & Fluoxetine & Phenytoin & Citalopram \\
\hline & & & Escitalopram \\
\hline & & & Sertraline \\
\hline & & & Clomipramine \\
\hline & & & Imipramine \\
\hline & & & Moclobemide \\
\hline & & & Antiepileptics \\
\hline & & & Phenytoin \\
\hline & & & Mephenytoin \\
\hline & & & Esobarbital \\
\hline & & & Mephobarbital \\
\hline & & & Phenobarbital \\
\hline & & & Primidone \\
\hline \multirow[t]{12}{*}{ CYP 2D6 } & Fluoxetine & & Antidepressants \\
\hline & Paroxetine & & Fluoxetine \\
\hline & Sertraline & & Fluvoxamine \\
\hline & Secondary TCA's & & Citalopram \\
\hline & & & Escitalopram \\
\hline & & & Duloxetine \\
\hline & & & Paroxetine \\
\hline & & & Venlafaxine \\
\hline & & & Trazodone \\
\hline & & & Maprotiline \\
\hline & & & Mirtazepine \\
\hline & & & TCA's \\
\hline \multirow[t]{11}{*}{ CYP 3A4 } & Fluoxetine & Carbamazepine & Antidepressants \\
\hline & Fluvoxamine & Barbiturates & Sertraline \\
\hline & Mirtazepine & Phenytoin & Venlafaxine \\
\hline & & St. John's Wort & Escitalopram \\
\hline & & & Mirtazepine \\
\hline & & & Trazodone \\
\hline & & & TCA's \\
\hline & & & Antiepileptics \\
\hline & & & Carbamazepine \\
\hline & & & Zonisamide \\
\hline & & & Tigabine \\
\hline
\end{tabular}

nomifensine was superior to amitriptyline. Overall, data about TCA's efficacy in the treatment of depression in epilepsy is largely uncontrolled or anecdotal but appears to support efficacy and tolerability [36].

SSRI's have become the first line of pharmacotherapy in most depressive disorders due to their proven efficacy and benign side-effect profiles. However, efficacy in epilepsy specifically has not been well studied yet. A series of open label studies support some efficacy and tolerability for sertraline, citalopram, mirtazepine, and fluoxetine. In general, SSRI's seem to be effective and well tolerated, but the response rates have been quite mixed across studies, likely due to great variability in sample populations, limited control of comorbid psychiatric disorders, and the occasional presence of cognitive disorders or brain damage in the samples [36].

However, Kühn et al. [108] produced a prospective study of safety and efficacy of citalopram, mirtazepine, and reboxetine (not available in North America) in TLE patients. They performed a post hoc analysis of 75 TLE patients with depression who received standard treatment with one of the above drugs. In general, they found that all the antidepressants were effective in treating the symptoms of depression, and that there were no serious adverse events or drug interactions. However, the dropout rate was significantly higher for mirtazepine that the other two agents, perhaps again because of the tendency of this drug to cause sedation, increased appetite, and weight gain in a population already often prone to these complications secondary to AED's.

8.2. Lithium. Although most known for its efficacy in bipolar disorders, lithium is also used as an augmentation strategy for treatment resistant unipolar depression. Unfortunately, even less has been published on the safety and efficacy of lithium in epilepsy than for antidepressants [36].

Coadministration of lithium carbonate and carbamazepine may have a benefit in terms of mood stabilization but appears to be associated with multiple interactions, including hematologic, thyroid, and electrolyte disregulation. However, lithium appears to be relatively tolerable when administered with valproate, but once again the additive aspects of sedation, weight gain, and tremor were noted. Lamotrigine and lithium appeared to be well tolerated together, though topiramate and lithium was associated with toxicity in at least one case [36].

Lithium also is known to be proconvulsive at higher doses, but this does not seem to be a significant concern at lower doses in epilepsy patients on AED's. However, as lithium is usually administered as an augmentation agent in unipolar depression, the additive risk of serotonin syndrome and lowered seizure threshold of both an antidepressant and lithium being administered to an epilepsy patient must be considered [36].

8.3. Psychotherapy. Once again, very little data has been collected on psychological therapies in the epilepsy population, let alone TLE. However, the few studies that exist seem to support the efficacy of cognitive behavioral therapies as useful in the treatment of depression in epilepsy [69]. 


\section{References}

[1] D. Kondziella, S. Alvestad, A. Vaaler, and U. Sonnewald, "Which clinical and experimental data link temporal lobe epilepsy with depression?" Journal of Neurochemistry, vol. 103, no. 6, pp. 2136-2152, 2007.

[2] W. A. M. Swinkels, J. Kuyk, R. van Dyck, and P. Spinhoven, "Psychiatric comorbidity in epilepsy," Epilepsy \& Behavior, vol. 7, no. 1, pp. 37-50, 2005.

[3] D. A. Pond and B. H. Bidwell, "A survey of epilepsy in fourteen general practices: II. Social and psychological aspects," Epilepsia, vol. 1, pp. 285-299, 1960.

[4] O. Gureje, "Interictal psychopathology in epilepsy: prevalence and pattern in a Nigerian clinic," The British Journal of Psychiatry, vol. 158, pp. 700-705, 1991.

[5] F. A. Gibbs, E. L. Gibbs, and B. Furster, "Psychomotor epilepsy," Archives of Neurology and Psychiatry, vol. 60, pp. 331-339, 1948.

[6] G. Gudmundsson, "Epilepsy in Iceland," Acta Neurologica Scandinavica, vol. 43, supplement 25, pp. 1-124, 1966.

[7] E. A. Rodin, M. Katz, and K. Lennox, "Differences between patients with temporal lobe seizures and those with other forms of epileptic attacks," Epilepsia, vol. 17, no. 3, pp. 313320, 1976.

[8] C. L. Harden, "The co-morbidity of depression and epilepsy: epidemiology, etiology, and treatment," Neurology, vol. 59, no. 6, supplement 4, pp. S48-S55, 2002.

[9] R. Manchanda, B. Schaefer, R. S. McLachlan, and W. T. Blume, "Interictal psychiatric morbidity and focus of epilepsy in treatment refractory patients admitted to an epilepsy unit," The American Journal of Psychiatry, vol. 149, no. 8, pp. 1096-1098, 1992.

[10] W. A. M. Swinkels, J. Kuyk, E. H. De Graaf, R. van Dyck, and P. Spinhoven, "Prevalence of psychopathology in Dutch epilepsy inpatients: a comparative study," Epilepsy \& Behavior, vol. 2, no. 5, pp. 441-447, 2001.

[11] J. G. Small, I. F. Small, and M. P. Hayden, "Further psychiatric investigations of patients with temporal and nontemporal lobe epilepsy," The American Journal of Psychiatry, vol. 123, no. 3, pp. 303-310, 1966.

[12] J. R. Stevens, "Psychiatric implications of psychomotor epilepsy," Archives of General Psychiatry, vol. 14, no. 5, pp. 461471, 1966.

[13] R. J. Mignone, E. F. Donnelly, and D. Sadowsky, "Psychological and neurological comparisons of psychomotor and nonpsychomotor epileptic patients," Epilepsia, vol. 11, no. 4, pp. 345-359, 1970.

[14] K. F. Standage and G. W. Fenton, "Psychiatric symptom profiles of patients with epilepsy: a controlled investigation," Psychological Medicine, vol. 5, no. 2, pp. 152-160, 1975.

[15] S. J. Adams, T. J. O’Brien, J. Lloyd, C. J. Kilpatrick, M. R. Salzberg, and D. Velakoulis, "Neuropsychiatric morbidity in focal epilepsy," The British Journal of Psychiatry, vol. 192, no. 6, pp. 464-469, 2008.

[16] V. Sanchez-Gistau, L. Pintor, G. Sugranyes et al., “Prevalence of interictal psychiatric disorders in patients with refractory temporal and extratemporal lobe epilepsy in Spain. A comparative study," Epilepsia, vol. 51, no. 7, pp. 1309-1313, 2010.

[17] W. A. M. Swinkels, W. van Emde Boas, J. Kuyk, R. Van Dyck, and P. Spinhoven, "Interictal depression, anxiety, personality traits, and psychological dissociation in patients with temporal lobe epilepsy (TLE) and extra-TLE," Epilepsia, vol. 47, no. 12, pp. 2092-2103, 2006.
[18] B. P. Hermann, S. Dikmen, and A. J. Wilensky, "Increased psychopathology associated with multiple seizure types: fact or artifact?" Epilepsia, vol. 23, no. 6, pp. 587-596, 1982.

[19] C. B. Dodrill, "Number of seizure types in relation to emotional and psychological adjustment in epilepsy," in Advances in Epileptology, R. J. Porter, A. A. Ward Jr., R. H. Mattson, and M. Dam, Eds., Epilepsy International Symposium, pp. 541544, Raven Press, New York, NY, USA, XVth edition, 1984.

[20] J. R. Stevens and B. P. Hermann, "Temporal lobe epilepsy, psychopathology, and violence: the state of the evidence," Neurology, vol. 31, no. 9, pp. 1127-1132, 1981.

[21] B. P. Hermann and S. Whitman, "Behavioral and personality correlates of epilepsy: a review, methodological critique, and conceptual model," Psychological Bulletin, vol. 95, no. 3, pp. 451-497, 1984.

[22] M. M. Robertson, S. Channon, and J. Baker, "Depressive symptomatology in a general hospital sample of outpatients with temporal lobe epilepsy: a controlled study," Epilepsia, vol. 35, no. 4, pp. 771-777, 1994.

[23] A. Indaco, P. B. Carrieri, C. Nappi, S. Gentile, and S. Striano, "Interictal depression in epilepsy," Epilepsy Research, vol. 12, no. 1, pp. 45-50, 1992.

[24] D. C. Hesdorffer, W. A. Hauser, J. F. Annegers, and G. Cascino, "Major depression is a risk factor for seizures in older adults," Annals of Neurology, vol. 47, no. 2, pp. 246-249, 2000.

[25] R. Reilly, S. Bowden, F. Bardenhagen, and M. Cook, "Equality of the psychological model underlying depressive symptoms in patients with temporal lobe epilepsy versus heterogeneous neurological disorders," Journal of Clinical and Experimental Neuropsychology, vol. 28, no. 7, pp. 1257-1271, 2006.

[26] D. Blumer, S. Wakhlu, K. Davies, and B. Hermann, "Psychiatric outcome of temporal lobectomy for epilepsy: incidence and treatment of psychiatric complications," Epilepsia, vol. 39, no. 5, pp. 478-486, 1998.

[27] O. Devinsky and S. Najjar, "Evidence against the existence of a temporal lobe personality syndrome," Neurology, vol. 53, no. 2, pp. 513-525, 1999.

[28] D. M. Bear and P. Fedio, "Quantitative analysis of interictal behavior in temporal lobe epilespy," Archives of Neurology, vol. 34, no. 8, pp. 454-467, 1977.

[29] D. Blumer, "Evidence supporting the temporal lobe epilepsy personality syndrome," Neurology, vol. 53, no. 2, pp. S9-S12, 1999.

[30] A. M. Kanner, A. M. Kozak, and M. Frey, "The use of sertraline in patients with epilepsy: is it safe?" Epilepsy \& Behavior, vol. 1, no. 2, pp. 100-105, 2000.

[31] A. J. Carson, A. Zemen, and M. C. Sharpe, "Neurology and neurosurgery," in Textbook of Psychosomatic Medicine, J. L. Levenson, Ed., chapter 32, pp. 759-795, American Psychiatric Publishing, Washington, DC, USA, 2nd edition, 2011.

[32] J. J. Barry, A. Lembke, and N. Huynh, "Affective Disorders in epilepsy," in Psychiatric Issues in Epilepsy: A Practical Guide to Diagnosis and Treatment, A. B. Ettinger and A. M. Kanner, Eds., pp. 45-71, Williams \& Wilkins, Philadelphia, Pa, USA, 2001.

[33] A. M. Kanner and J. C. R. Nieto, "Depressive disorders in epilepsy," Neurology, vol. 53, no. 2, pp. S26-S32, 1999.

[34] W. A. Lishman, Organic Psychiatry, Blackwell Scientific Publications, Oxford, UK, 3rd edition, 1998.

[35] J. E. Jones, B. P. Hermann, J. J. Barry, F. Gilliam, A. M. Kanner, and K. J. Meador, "Clinical Assessment of Axis I psychiatric morbidity in chronic epilepsy: a multicenter investigation," 
The Journal of Neuropsychiatry and Clinical Neurosciences, vol. 17, no. 2, pp. 172-179, 2005.

[36] M. Mula, B. Schmitz, and J. W. Sander, "The pharmacological treatment of depression in adults with epilepsy," Expert Opinion on Pharmacotherapy, vol. 9, no. 18, pp. 3159-3168, 2008.

[37] Y. I. Sheline, "Neuroimaging studies of mood disorder effects on the brain," Biological Psychiatry, vol. 54, no. 3, pp. 338352, 2003.

[38] B. S. McEwen, "Glucocorticoids, depression, and mood disorders: structural remodeling in the brain," Metabolism, vol. 54, no. 5, pp. S20-S23, 2005.

[39] H. Lavretsky, M. Ballmaier, D. Pham, A. Toga, and A. Kumar, "Neuroanatomical characteristics of geriatric apathy and depression: a magnetic resonance imaging study," The American Journal of Geriatric Psychiatry, vol. 15, no. 5, pp. 386-394, 2007.

[40] S. G. Mueller, K. D. Laxer, N. Schuff, and M. W. Weiner, "Voxel-based T2 relaxation rate measurements in temporal lobe epilepsy (TLE) with and without mesial temporal sclerosis," Epilepsia, vol. 48, no. 2, pp. 220-228, 2007.

[41] J. D. Bremner, M. Narayan, E. R. Anderson, L. H. Staib, H. L. Miller, and D. S. Charney, "Hippocampal volume reduction in major depression," The American Journal of Psychiatry, vol. 152, pp. 973-981, 2000.

[42] G. D. Cascino, C. R. Jack Jr., J. E. Parisi et al., "Magnetic resonance imaging based volume studies in temporal lobe epilepsy: pathological correlations," Annals of Neurology, vol. 30, no. 1, pp. 31-36, 1991.

[43] Y. I. Sheline, P. W. Wang, M. H. Gado, J. G. Csernansky, and M. W. Vannier, "Hippocampal atrophy in recurrent major depression," Proceedings of the National Academy of Sciences of the United States of America, vol. 93, no. 9, pp. 3908-3913, 1996.

[44] Y. I. Sheline, M. Sanghavi, M. A. Mintun, and M. H. Gado, "Depression duration but not age predicts hippocampal volume loss in medically healthy women with recurrent major depression," Journal of Neuroscience, vol. 19, no. 12, pp. 5034 5043, 1999.

[45] S. A. Baxendale, P. J. Thompson, and J. S. Duncan, "Epilepsy and depression: the effects of comorbidity on hippocampal volume-a pilot study," Seizure, vol. 14, no. 6, pp. 435-438, 2005.

[46] M. F. Dulay, B. K. Schefft, J. D. Fargo, M. D. Privitera, and H. S. Yeh, "Severity of depressive symptoms, hippocampal sclerosis, auditory memory, and side of seizure focus in temporal lobe epilepsy," Epilepsy \& Behavior, vol. 5, no. 4, pp. 522-531, 2004.

[47] C. Helmstaedter, M. Sonntag-Dillender, C. Hoppe, and C. E. Elger, "Depressed mood and memory impairment in temporal lobe epilepsy as a funtion of focus lateralization and localization," Epilepsy \& Behavior, vol. 5, no. 5, pp. 696-701, 2004.

[48] E. A. Phelps and J. E. LeDoux, "Contributions of the amygdala to emotion processing: from animal models to human behavior," Neuron, vol. 48, no. 2, pp. 175-187, 2005.

[49] E. J. Richardson, H. R. Griffith, R. C. Martin et al., "Structural and functional neuroimaging correlates of depression in temporal lobe epilepsy," Epilepsy \& Behavior, vol. 10, no. 2, pp. 242-249, 2007.

[50] L. Tebartz Van Elst, F. G. Woermann, L. Lemieux, and M. R. Trimble, "Amygdala enlargement in dysthymia: a volumetric study of patients with temporal lobe epilepsy," Biological Psychiatry, vol. 46, no. 12, pp. 1614-1623, 1999.
[51] A. Quiske, C. Helmstaedter, S. Lux, and C. E. Elger, "Depression in patients with temporal lobe epilepsy is related to mesial temporal sclerosis," Epilepsy Research, vol. 39, no. 2, pp. 121-125, 2000.

[52] M. Mula, M. R. Trimble, and J. W. A. S. Sander, "The role of hippocampal sclerosis in topiramate-related depression and cognitive deficits in people with epilepsy," Epilepsia, vol. 44, no. 12, pp. 1573-1577, 2003.

[53] P. C. B. Salgado, C. L. Yasuda, and F. Cendes, "Neuroimaging changes in mesial temporal lobe epilepsy are magnified in the presence of depression," Epilepsy \& Behavior, vol. 19, no. 3, pp. 422-427, 2010.

[54] J. Foong and D. Flugel, "Psychiatric outcome of surgery for temporal lobe epilepsy and presurgical considerations," Epilepsy Research, vol. 75, no. 2-3, pp. 84-96, 2007.

[55] L. Altshuler, R. Rausch, S. Delrahim, J. Kay, and P. Crandall, "Temporal lobe epilepsy, temporal lobectomy, and major depression," Journal of Neuropsychiatry and Clinical Neurosciences, vol. 11, no. 4, pp. 436-443, 1999.

[56] K. Malmgren, J. E. Starmark, G. Ekstedt, H. Rosén, and C. Sjöberg-Larsson, "Nonorganic and organic psychiatric disorders in patients after epilepsy surgery," Epilepsy \& Behavior, vol. 3, no. 1, pp. 67-75, 2002.

[57] M. Quigg, D. K. Broshek, S. Heidal-Schiltz, J. W. Maedgen, and E. H. Bertram III, "Depression in intractable partial epilepsy varies by laterality of focus and surgery," Epilepsia, vol. 44, no. 3, pp. 419-424, 2003.

[58] M. Reuber, B. Andersen, C. E. Elger, and C. Helmstaedter, "Depression and anxiety before and after temporal lobe epilepsy surgery," Seizure, vol. 13, no. 2, pp. 129-135, 2004.

[59] H. A. Ring, J. Moriarty, and M. R. Trimble, "A prospective study of the early postsurgical psychiatric associations of epilepsy surgery," Journal of Neurology Neurosurgery and Psychiatry, vol. 64, no. 5, pp. 601-604, 1998.

[60] J. Wrench, S. J. Wilson, and P. F. Bladin, "Mood disturbance before and after seizure surgery: a comparison of temporal and extratemporal resections," Epilepsia, vol. 45, no. 5, pp. 534-543, 2004.

[61] P. A. Derry, K. J. Rose, and R. S. McLachlan, "Moderators of the effect of preoperative emotional adjustment on postoperative depression after surgery for temporal lobe epilepsy," Epilepsia, vol. 41, no. 2, pp. 177-185, 2000.

[62] Y. Inoue and T. Mihara, "Psychiatric disorders before and after surgery for epilepsy," Epilepsia, vol. 42, no. 8, pp. 13-18, 2001.

[63] G. N. Meldolesi, G. Di Gennaro, P. P. Quarato et al., "Changes in depression, anxiety, anger, and personality after resective surgery for drug-resistant temporal lobe epilepsy: a 2 year follow up study," Epilepsy Research, vol. 77, no. 1, pp. 22-30, 2007.

[64] G. Glosser, A. S. Zwil, D. S. Glosser, M. J. O’Connor, and M. R. Sperling, "Psychiatric aspects of temporal lobe epilepsy before and after anterior temporal lobectomy," Journal of Neurology Neurosurgery and Psychiatry, vol. 68, no. 1, pp. 53$58,2000$.

[65] E. Irle, M. Peper, B. Wowra, and S. Kunze, "Mood changes after surgery for tumors of the cerebral cortex," Archives of Neurology, vol. 51, no. 2, pp. 164-174, 1994.

[66] A. M. Pringle, R. Taylor, and I. R. Whittle, "Anxiety and depression in patients with an intracranial neoplasm before and after tumour surgery," The British Journal of Neurosurgery, vol. 13, no. 1, pp. 46-51, 1999.

[67] B. Metternich, K. Wagner, A. Brandt et al., "Preoperative depressive symptoms predict postoperative seizure outcome in 
temporal and frontal lobe epilepsy," Epilepsy \& Behavior, vol. 16, no. 4, pp. 622-628, 2009.

[68] J. M. Wrench, G. Rayner, and S. J. Wilson, "Profiling the evolution of depression after epilepsy surgery," Epilepsia, vol. 52, no. 5, pp. 900-908, 2011.

[69] M. Mula and B. Schmitz, "Depression in epilepsy: mechanisms and therapeutic approach," Therapeutic Advances in Neurological Disorders, vol. 2, no. 5, pp. 337-344, 2009.

[70] C. L. Melvin, T. S. Carey, F. Goodman, J. M. Oldham, J. W. Williams, and L. M. Ranney, "Effectiveness of antiepileptic drugs for the treatment of bipolar disorder: findings from a systematic review," Journal of Psychiatric Practice, vol. 14, supplement 1, pp. 9-14, 2008.

[71] M. Mula, S. Pini, and G. B. Cassano, "The role of anticonvulsant drugs in anxiety disorders: a critical review of the evidence," Journal of Clinical Psychopharmacology, vol. 27, no. 3, pp. 263-272, 2007.

[72] D. F. Zullino, Y. Khazaal, J. Höttenschwiler, F. Borgeat, and J. Besson, "Anticonvulsant drugs in the treatment of substance withdrawal," Drugs of Today, vol. 40, no. 7, pp. 603-619, 2004.

[73] A. D. Lopez, "The global burden of disease 1990-2020. World Health Organization," in Proceedings of the International Seminar on Nuclear War and Planetary Emergencies, 25th session, pp. 49-50.

[74] L. Forsgren and L. Nystrom, "An incident case-referent study of epileptic seizures in adults," Epilepsy Research, vol. 6, no. 1, pp. 66-81, 1990.

[75] D. C. Hesdorffer, W. Allen Hauser, E. Olafsson, P. Ludvigsson, and O. Kjartansson, "Depression and suicide attempt as risk factors for incident unprovoked seizures," Annals of Neurology, vol. 59, no. 1, pp. 35-41, 2006.

[76] J. K. Austin, J. Harezlak, D. W. Dunn, G. A. Huster, D. F. Rose, and W. T. Ambrosius, "Behavior problems in children before first recognized seizures," Pediatrics, vol. 107, no. 1, pp. 115$122,2001$.

[77] A. T. McAfee, K. E. Chilcott, C. B. Johannes, K. Hornbuckle, W. A. Hauser, and A. M. Walker, "The incident of first unprovoked seizure in pediatric patients with and without psychiatric distress," Epilepsia, vol. 48, no. 6, pp. 1075-1082, 2007.

[78] K. Alper, K. A. Schwartz, R. L. Kolts, and A. Khan, "Seizure incidence in psychopharmacological clinical trials: an analysis of Food \& Drug Administration (FDA) summary basis of approval reports," Biological Psychiatry, vol. 62, no. 4, pp. 345-354, 2007.

[79] J. Christensen, M. Vestergaard, P. B. Mortensen, P. Sidenius, and E. Agerbo, "Epilepsy and risk of suicide: a population based case control study," The Lancet Neurology, vol. 6, no. 8, pp. 693-698, 2007.

[80] J. Christensen, M. Vestergaard, M. C. Petersen, and P. Sidenius, "Epilepsy in Denmark: incidence, prevalence, and validation of diagnosis," Epilepsia, vol. 46, supplement 6, p. 386, 2005.

[81] D. Blumer, G. Montouris, K. Davies, A. Wyler, B. Phillips, and B. Hermann, "Suicide in epilepsy: psychopathology, pathogenesis, and prevention," Epilepsy \& Behavior, vol. 3, no. 3, pp. 232-241, 2002.

[82] K. A. Miczek, E. W. Fish, J. F. de Bold, and R. M. M. de Almedia, "Social and neural determnants of aggressive behavior: pharmacotherapeutic targets at serotonin, dopamine, and gamma aminobutyric acid systems," Psychopharmacology, vol. 163, pp. 434-458, 2002.

[83] M. Pompili, P. Girardi, A. Ruberto, and R. Tatarelli, "Suicide in the epilepsies: a meta-analytic investigation of 29 cohorts," Epilepsy \& Behavior, vol. 7, no. 2, pp. 305-310, 2005.
[84] J. G. Keilp, H. A. Sackeim, B. S. Brodsky, and M. A. Oquendo, "Prevention of suicide and attempted suicide in Denmark: epidemiological studies of suicide and interventional studies in selected risk groups," Danish Medical Bulletin, vol. 54, no. 4, pp. 306-359, 2007.

[85] J. G. Keilp, H. A. Sackeim, B. S. Brodsky, M. A. Oquendo, K. M. Malone, and J. J. Mann, "Neuropsychological dysfunction in depressed suicide attempters," The American Journal of Psychiatry, vol. 158, no. 5, pp. 735-741, 2001.

[86] A. Raust, F. Slama, F. Mathieu et al., "Prefrontal cortex dysfunction in patients with suicidal behavior," Psychological Medicine, vol. 37, no. 3, pp. 411-419, 2007.

[87] J. E. Jones, B. P. Hermann, J. J. Barry, F. G. Gilliam, A. M. Kanner, and K. J. Meador, "Rates and risk factors for suicide, suicidal ideation, and suicide attempts in chronic epilepsy," Epilepsy \& Behavior, vol. 4, no. 3, pp. S31-S38, 2003.

[88] A. G. Espinosa, R. A. Machado, S. B. González, M. E. G. González, A. P. Montoto, and G. T. Sotomayor, "Wisconsin card sorting test performance and impulsivity in patients with temporal lobe epilepsy: suicidal risk and suicide attempts," Epilepsy \& Behavior, vol. 17, no. 1, pp. 39-45, 2010.

[89] Food \& Drug Administration, Office of Biostatistics, "Statistical review and evaluation: antiepileptic drugs and suicidality," 2008, http://www.fda.gov/ohrms/dockets/ac/08/. briefing/2008-4372b1-01-FDA.pdf.

[90] X. Wen, K. J. Meador, D. W. Loring, S. Eisenschenk, R. Segal, and A. G. Hartzema, "Is antiepileptic drug use related to depression and suicidal ideation among patients with epilepsy?" Epilepsy \& Behavior, vol. 19, no. 3, pp. 494-500, 2010.

[91] J. Lehrner, R. Kalchmayr, W. Serles et al., "Health related quality of life (HRQOL), activity of daily living (ADL) and depressive mood disorder in temporal lobe epilepsy," Seizure, vol. 8, no. 2, pp. 88-92, 1999.

[92] K. Perrine, B. P. Hermann, K. J. Meador et al., "The relationship of neuropsychological functioning to quality of life in epilepsy," Archives of Neurology, vol. 52, no. 10, pp. 997-1003, 1995.

[93] F. Gilliam, R. Kuzniecky, E. Faught, L. Black, G. Carpenter, and R. Schrodt, "Patient validated content of epilepsy-specific quality of life measurements," Epilepsia, vol. 38, no. 2, pp. 233-236, 1997.

[94] L. S. Boylan, L. A. Flint, D. L. Labovitz, S. C. Jackson, K. Starner, and O. Devinsky, "Depression but not seizure frequency predicts quality of life in treatment resistant epilepsy," Neurology, vol. 62, no. 2, pp. 258-261, 2004.

[95] J. A. Cramer, D. Blum, M. Reed et al., "The influence of comorbid depression on quality of life for people with epilepsy," Epilepsy \& Behavior, vol. 4, no. 5, pp. 515-521, 2003.

[96] E. K. Johnson, J. E. Jones, M. Seidenberg, and B. P. Hermann, "The relative impact of anxiety, depression, and clinical seizure features on health-related quality of life in epilepsy," Epilepsia, vol. 45, no. 5, pp. 544-550, 2004.

[97] A. M. Kanner, F. G. Gilliam, B. Hermann, and K. J. Meador, "Differential effect of mood and anxiety disorders on the quality of life and perception of adverse events to antiepileptic drugs in patients with epilepsy. In: abstracts from the 2007 Annual Meeting of the American Epilepsy Society," Epilepsia, vol. 48, 6, pp. 1-118, 2007.

[98] G. N. Meldolesi, A. Picardi, P. P. Quarato et al., "Factors associated with generic and disease specific quality of life in temporal love epilepsy," Epilepsy Research, vol. 69, no. 2, pp. 135-146, 2006.

[99] A. M. Kanner, "Psychiatric issues in epilepsy: the complex relation of mood, anxiety disorders, and epilepsy," Epilepsy \& Behavior, vol. 15, no. 1, pp. 83-87, 2009. 
[100] M. R. Salzberg and F. J. E. Vajda, "Epilepsy, depression and antidepressant drugs," Journal of Clinical Neuroscience, vol. 8, no. 3, pp. 209-215, 2001.

[101] J. D. Leander, "Fluoxetine, a selective serotonin-uptake inhibitor, enhances the anticonvulsant effects of phenytoin, carbamazepine, and ameltolide (LY201116)," Epilepsia, vol. 33, no. 3, pp. 573-576, 1992.

[102] E. Favale, V. Rubino, P. Mainardi, G. Lunardi, and C. Albano, "Anticonvulsant effect of fluoxetine in humans," Neurology, vol. 45, no. 10, pp. 1926-1927, 1995.

[103] C. Albano, A. Cupello, P. Mainardi, S. Scarrone, and E. Favale, "Successful treatment of epilepsy with serotonin reuptake inhibitors: proposed mechanism," Neurochemical Research, vol. 31, no. 4, pp. 509-514, 2006.

[104] M. Mula and M. R. Trimble, "Pharmacokinetic interactions between antiepileptic and antidepressant drugs," The World Journal of Biological Psychiatry, vol. 4, no. 1, pp. 21-24, 2003.

[105] M. Mula and B. Schmitz, "Depression in epilepsy-mechanisms and therapeutic approaches," European Neurological Disorder, vol. 2, pp. 58-60, 2007.

[106] J. Szymura-Oleksiak, E. Wyska, and A. Wasieczko, "Pharmacokinetic interaction between imipramine and carbamazepine in patients with major depression," Psychopharmacology, vol. 154, no. 1, pp. 38-42, 2001.

[107] C. Fehr, G. Grunder, C. Hiemke, and N. Dahmen, "Increase in serum clomipramine concentrations caused by valproate," Journal of Clinical Psychopharmacology, vol. 20, no. 4, pp. 493-494, 2000.

[108] K. U. Kühn, B. B. Quednow, M. Thiel, P. Falkai, W. Maier, and C. E. Elger, "Antidepressive treatment in patients with temporal lobe epilepsy and major depression: a prospective study with three different antidepressants," Epilepsy \& Behavior, vol. 4, no. 6, pp. 674-679, 2003. 


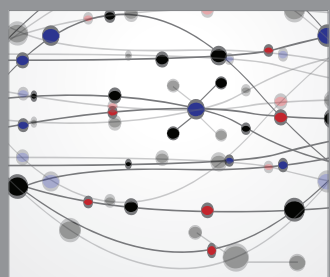

The Scientific World Journal
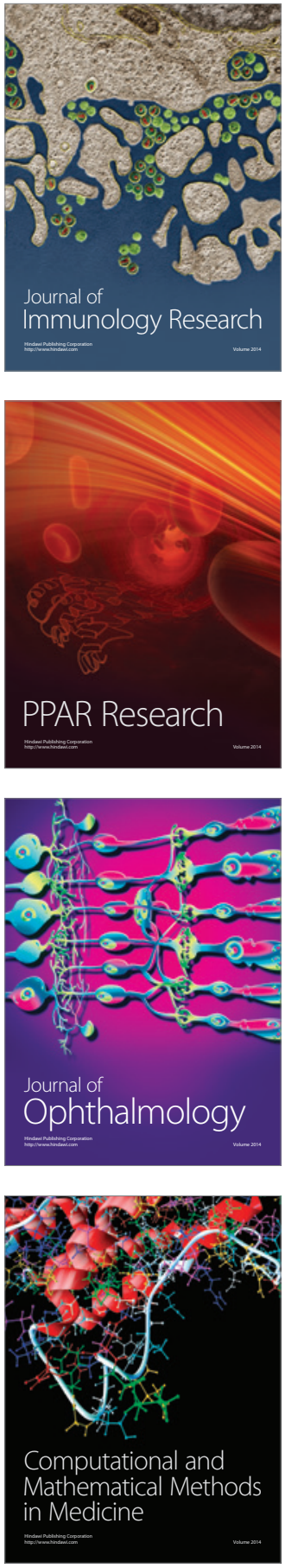

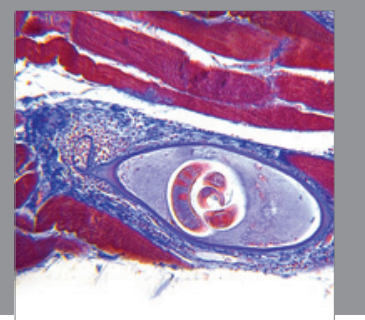

Gastroenterology

Research and Practice
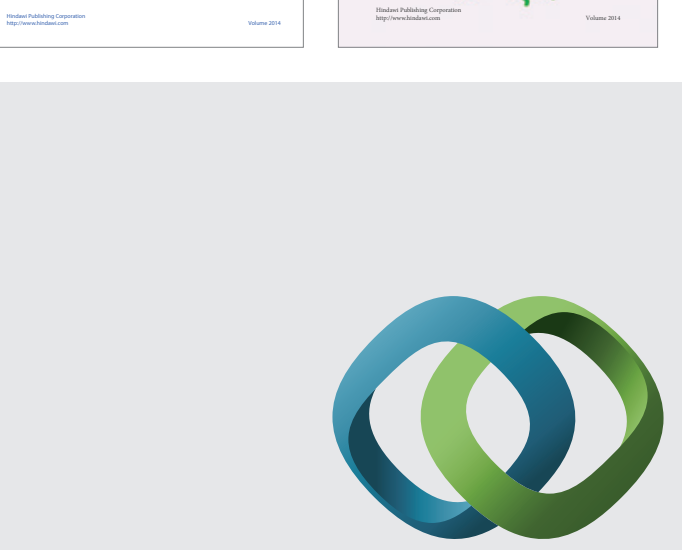

\section{Hindawi}

Submit your manuscripts at

http://www.hindawi.com
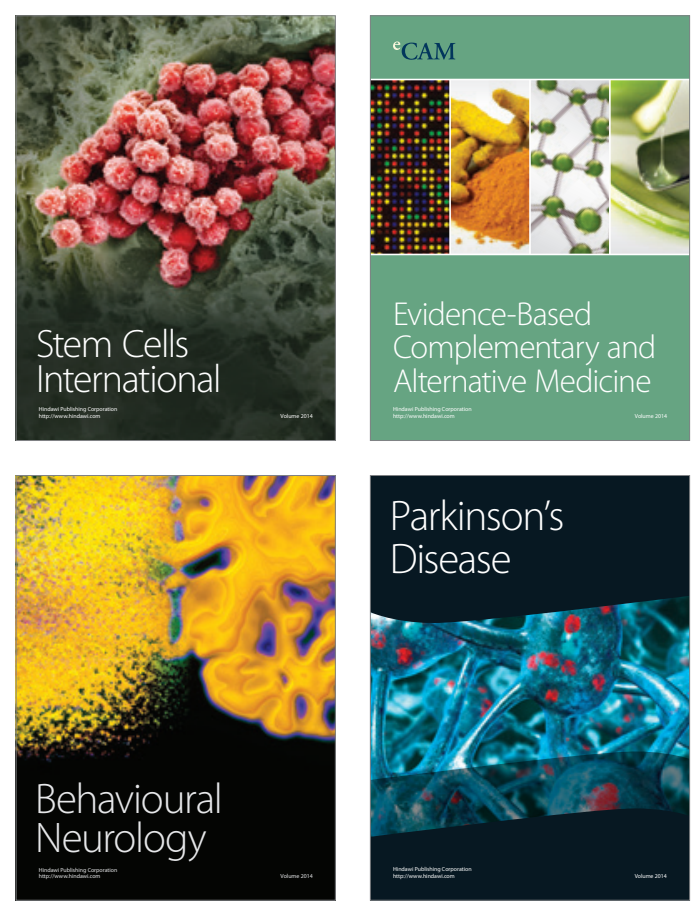

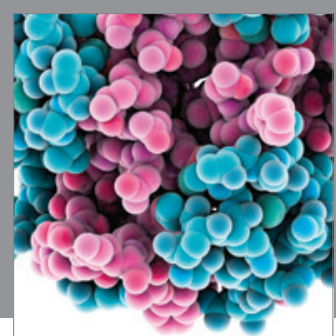

Journal of
Diabetes Research

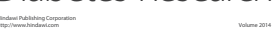

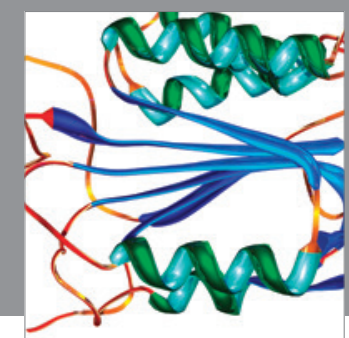

Disease Markers
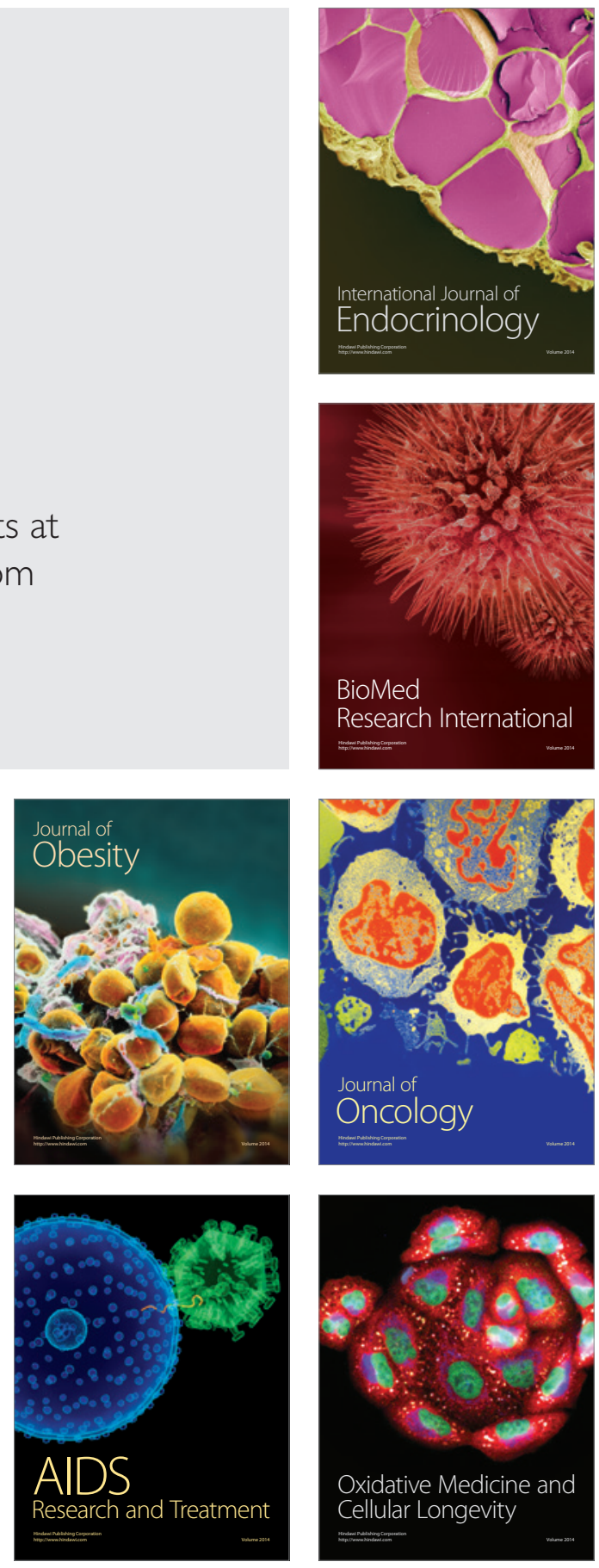